\title{
Aperture Coupled Dielectric Resonator Antenna Embedded in a Secondary Substrate for Mechanical Firmness
}

\author{
Suparna BALLAV, Susanta Kumar PARUI \\ Dept. of Electronics \& Tele-comm. Engineering, Indian Institute of Science Engineering and Technology, Shibpur, \\ Howrah-711103, India \\ ballav.suparna@gmail.com, arkapv@yahoo.com \\ Submitted February 15, 2018 / Accepted April 29, 2018
}

\begin{abstract}
An aperture coupled rectangular dielectric resonator antenna (RDRA) is proposed where high permittivity radiating element is embedded on a comparatively low permittivity secondary substrate material (here FR4 is used). Proposed dielectric resonator antenna (DRA) is accurately excited in fundamental mode $T E_{111}$ for efficient broadside radiation. All the existing DRAs are placed on ground plane using adhesive which is not suitable for practical application. The proposed design can be considered as a promising solution of mounting of DRA on the ground plane with good mechanical firmness. Since the RDRA is embedded on FR4 substrate, it can be withstand very high pressure and vibrating condition which is the main practical limitation of existing DRA technology. The effect of embedding the radiating DRA on secondary substrate is studied here in details. A prototype of single antenna element is designed, fabricated and measured to validate the simulation results. Further, a $2 \times 2$ antenna array with such antenna element is designed at the center frequency of $6.684 \mathrm{GHz}$ for obtaining higher directive gain. The array offers an impedance bandwidth of $4.94 \%$ and a maximum gain of $13.4 \mathrm{dBi}$ at the operating frequency. A good agreement has been obtained between the measured and simulated results.
\end{abstract}

\section{Keywords}

Aperture coupled, antenna gain, dielectric resonator antenna (DRA), embedded

\section{Introduction}

The versatility in design offered by dielectric resonator antennas (DRAs) make them attractive alternatives to other low-gain antennas such as microstrip antennas or wire antennas. Lack of surface wave losses and minimal conductor losses help them to maintain high radiation efficiency at microwaves or millimeter-waves frequencies. Various shapes of different dielectric materials can be used for design DRAs. Rectangular shapes of DRA are generally preferred over other basic shapes such as cylindrical or hemispherical due to their fabrication simplicity and additional degrees of freedom from designer aspect [1-3]. Again DRA supports different modes and each mode of DRA has its unique electromagnetic field distributions and hence different modes of DRA need different boundary conditions to be met [4-6]. As a consequence different feeding mechanism has to be used for individual modes to satisfy different boundary conditions. In literature several types of feeding mechanism for DRA are proposed, such as probe feed, microstrip line feed, slot aperture coupling, coplanar line feed, dielectric image guide coupling and so on [2]. Again the excitable DRA modes strongly depend on the position of feeding source. They are also the main decisive factor for determining nature of radiation pattern of DRA. In this paper, the aperture-coupled source [7] is preferred because the ground plane can isolate its feed network from the DRA and, thus, spurious radiation from the feed network can be avoided which ensure that most of the energy is radiated from DRA. Again it is easier to obtain a good single mode operation using a centrally fed slot because all modes TM and half of the TE modes are eliminated (for this configuration, only $\mathrm{TE}_{m n l}$ modes with odd $m, n, 1$ can be excited) [8]. Recently DRA arrays have found widespread attentions due to high gain, wide bandwidth, low side-lobe-level, high efficiency in various communication systems. But most of the DRAs are mounted over ground plane using adhesive or superglue [9-11]. This technique is not suitable for adverse conditions (high temperature, high pressure etc.). For good performance of array each individual DRA element must be placed and attached at the appropriate position. As we go for higher frequency, the size of the elements becomes small, and their exact locations become more decisive, in that situation this approach not suitable in practice. Aim of our study is to investigate whether a DRA embedded on a secondary substrate radiates efficiently or not which can eliminate the above mentioned practical limitations. Some works were done [12], [13] earlier where DRA's are embedded within one another to enhance impedance bandwidth. In [14-16], 
an array of DRAs has been designed from a single dielectric sheet with a lattice of holes using perforation technique. This technique of fabricating DRA arrays eliminates the requirement of positioning and bonding individual DRA elements in an array. But the design methodology of those lattice holes to get exact array performance is quite complex.

In this paper, a simple aperture-coupled RDRA is proposed where high permittivity radiating element is embedded on a secondary substrate of comparatively low permittivity than the radiating DRA (here FR4 is used as secondary substrate). The proposed DRA is accurately excited in fundamental mode $\mathrm{TE}_{111}$ for efficient radiation. The main feature of the proposed embedded RDRA is that it can be used in any adverse conditions (especially in high pressure and trembling environment). In the proposed design the radiation occurs mainly due to the RDRA. Secondary substrate i.e. Substrate2 helps to hold the DRA and hardly affects the resonance frequency of the antenna. So for large array design using DRA this embedded technique will become very handy. A comparative study is performed between a single element embedded DRA and normal DRA. Then we further extent our work to design DRA arrays. Performance of a $2 \times 2$ embedded array has been studied. The embedded array is operating at $6.684 \mathrm{GHz}$ with peak gain of $13.4 \mathrm{dBi}$ with an accurate broadside radiation pattern.

\subsection{The Configuration}

The configuration of the aperture-coupled RDRA with length $l$, width $w$, and height $h$ placed on a ground plane is shown in Fig. 1. It consists of a RDRA embedded on FR4 substrate and the feeding circuit is fabricated on Arlon AD270 substrate. A rectangular aperture is cut in the ground plane to couple the energy from the microstrip line to the dielectric resonators. A centrally fed aperture has been selected to excite the RDRA such that it radiates like a horizontal magnetic dipole. The RDRA has 3 dimensions (length, width, and height), which helps to determine the resonant frequency of a required modes. In this section, the dielectric wave guide model (DWM) is used to determine the resonant frequency of RDRA operating at fundamental mode for given dimension. Using the DWM model, the resonance frequency of the $\mathrm{TE}_{p q r}$ mode is given by [2]:

$$
f_{0}=\frac{c}{2 \pi \sqrt{\varepsilon_{\mathrm{r}}}} \sqrt{k_{\mathrm{x}}^{2}+k_{\mathrm{y}}^{2}+k_{\mathrm{z}}^{2}}
$$

where

$$
\begin{gathered}
k_{\mathrm{x}}^{2}+k_{\mathrm{y}}^{2}+k_{\mathrm{z}}^{2}=\varepsilon_{\mathrm{r}} k_{\mathrm{o}}^{2}, \\
k_{\mathrm{x}}=\frac{p \pi}{l} ; k_{\mathrm{z}}=\frac{r \pi}{2 h}, \\
w k_{\mathrm{y}}=q \pi-2 \arctan \left\{\frac{k_{\mathrm{y}}}{\sqrt{\left(\varepsilon_{\mathrm{r}}-1\right)} k_{\mathrm{o}}^{2}-k_{\mathrm{y}}^{2}}\right\}
\end{gathered}
$$

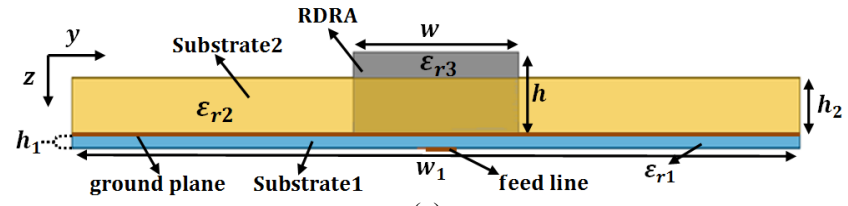

(a)

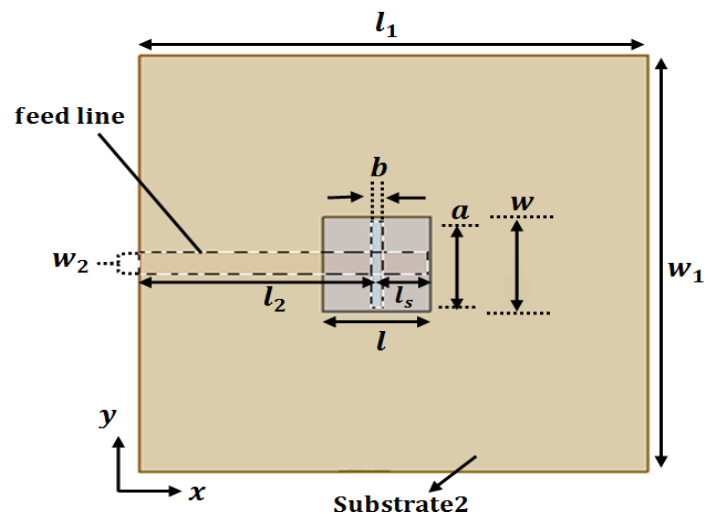

(b)

Fig. 1. Configuration of the proposed antenna: $l_{1}=45 \mathrm{~mm}$, $w_{1}=42 \mathrm{~mm}, l=9.925 \mathrm{~mm}, w=9.525 \mathrm{~mm}, h=4.6 \mathrm{~mm}$. (a) Side siew. (b) Top view.

in which $k_{\mathrm{x}}, k_{\mathrm{y}}$, and $k_{\mathrm{z}}$ are denoted as the wave numbers along the $x$-, $y$ - and $z$-directions respectively. $k_{\mathrm{o}}$ is the freespace wave number corresponding to the resonant frequency. All of the subscripts $p, q, r$ of $\mathrm{TE}_{p q r}$, must be odd numbers for a centrally fed rectangular aperture as boundary condition not permits those resonant modes. We have considered $\mathrm{TE}_{111}$ modes for our design purpose. By solving the transcendental equations given in (1-4) numerically using a programming code in Matlab, we have estimated the resonance frequency for $\mathrm{TE}_{111}$ mode. The initially the dimension of the aperture is model as [2]:

$$
\begin{gathered}
a=\frac{0.4 \lambda_{\mathrm{o}}}{\sqrt{\varepsilon_{\mathrm{e}}}}, \\
\varepsilon_{\mathrm{e}}=\frac{\varepsilon_{\mathrm{r} 1}+\varepsilon_{\mathrm{r} 3}}{2}, \\
b=0.2 a .
\end{gathered}
$$

For the center frequency $f_{\mathrm{o}}$ of operation the values become $a \approx 5.4 \mathrm{~mm}$ and $\mathrm{b} \approx 1.02 \mathrm{~mm}$. After optimization we have selected $a=9 \mathrm{~mm}$ and $b=1 \mathrm{~mm}$ which gives good impedance matching as well as properly excite the desired mode of the RDRA. A RDRA of dimensions $9.525 \mathrm{~mm} \times$ $9.525 \mathrm{~mm} \times 4.6 \mathrm{~mm}$ has been chosen to operate at the desired frequency and embedded on FR4 substrate of dielectric constant $\varepsilon_{\mathrm{r} 2}=4.4$, loss tangent of 0.02 and thickness of $3.16 \mathrm{~mm}$. Eccostock HIK material of dielectric constant $\varepsilon_{\mathrm{r} 3}=20$ has been used to fabricate the prototype of RDRA. The $50 \Omega$ microstrip feed line is designed on Arlon AD270 substrate with the thickness of $0.79 \mathrm{~mm}$, loss tangent of 0.0023 and dielectric constant $\varepsilon_{\mathrm{r} 1}$ of 2.7.The length of the microstrip line is varied and minimum $\mathrm{S}_{11}$ has obtained for $l_{\mathrm{s}}=4.5 \mathrm{~mm}$ as shown in Fig. 2. All simulations have been performed using FEM based High-Frequency Structure Simulator (HFSS). 


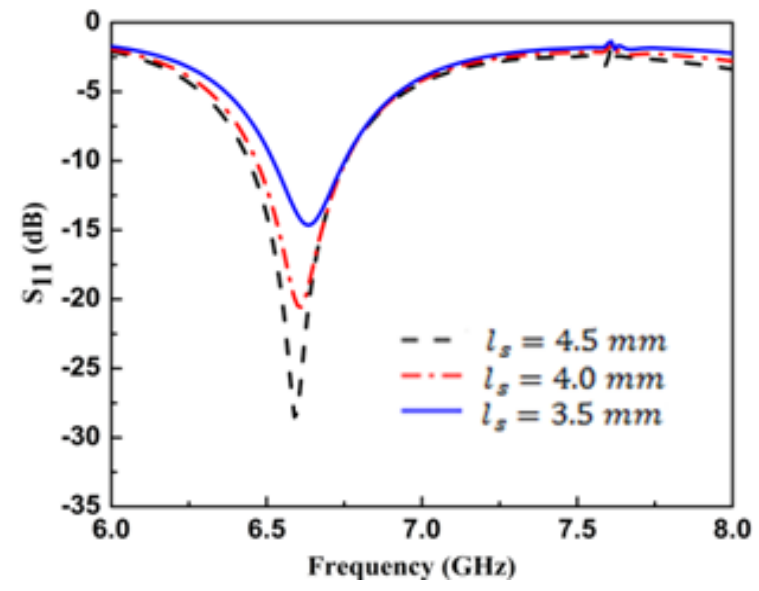

Fig. 2. Simulated $S_{11}$ of the single element RDRA for different values of $l_{\mathrm{s}}$.

\subsection{Simulated and Measured Results}

Figure 3 shows the fabricated prototype of single element embedded RDRA. The simulated and measured $\mathrm{S}_{11}$ of the prototype is shown in Fig. 4 with reasonable agreement between simulation and experimental data. As can be shown from Fig. 4 the RDRA element operates at $6.7 \mathrm{GHz}$ with $5.6 \%-10 \mathrm{~dB}$ impedance bandwidth. An Agilent N5230A vector network analyzer is used to measure the S-parameters. The simulated electric field distribution at $6.59 \mathrm{GHz}$ is matched with the ideal E-field distributions due to $\mathrm{TE}_{111}$ mode as shown in Fig. 5.

E-field distribution over RDRA and secondary substrate FR4 is shown in Fig. 6 at resonance frequency $6.59 \mathrm{GHz}$. From the field distribution we can easily conclude that most of the fields is confined within the RDRA hence losses due the secondary FR4 substrate are negotiable. The simulated and measured E-plane (xz plane) and Hplane (yz plane) normalized radiation patterns at $6.7 \mathrm{GHz}$ are shown in Fig. 7 and Fig. 8, respectively. The measured E-plane and $\mathrm{H}$-plane peak gains are $3.2 \mathrm{dBi}$ and $3.7 \mathrm{dBi}$, respectively. The measured cross-polarization levels for both E-plane and H-plane are very less from their peak values.

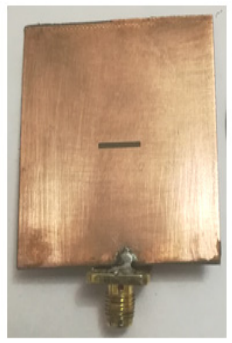

(a)

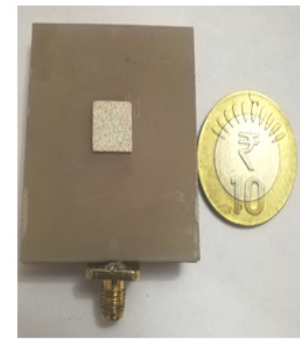

(b)

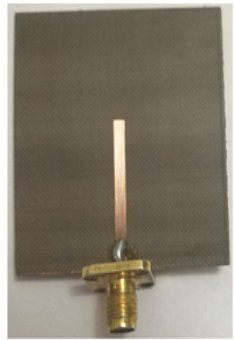

(c)
Fig. 3. Fabricated prototype of single element embedded RDRA. (a) Perspective view of the aperture on the ground plane. (b) Perspective view of the antenna. (c) Perspective view of the feed line below the ground plane.

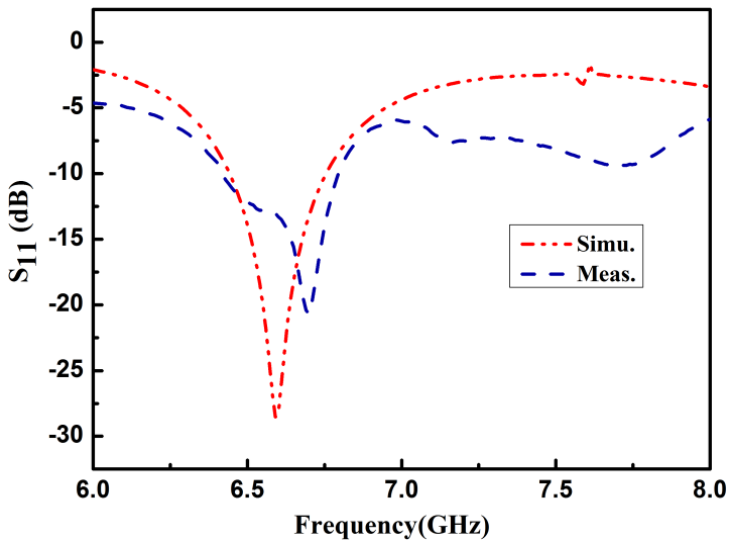

Fig. 4. Simulated and measured $S_{11}$ of the single element RDRA embedded on FR4 substrate. The parameters are shown as in Fig. 1.

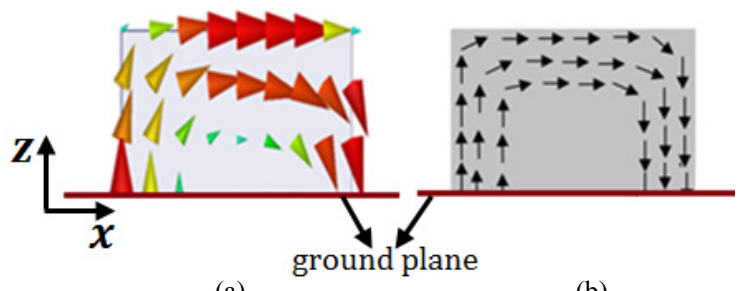

(a)

(b)

Fig. 5. (a) Full-wave simulated vector E-field of $\mathrm{TE}_{111}$ mode at minimum $6.59 \mathrm{GHz}$ in the single element RDRA. Red (colored diagram): high-intensity field. (b) Ideal E-field distributions in RDRA due to $\mathrm{TE}_{111}$ mode

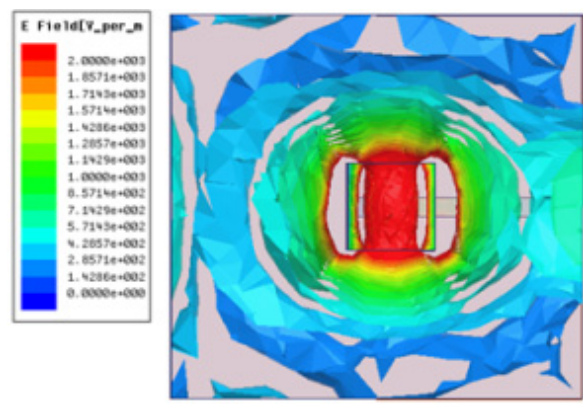

Fig. 6. Full-wave simulated E-fields distribution over the whole antenna at $f=6.59 \mathrm{GHz}$.

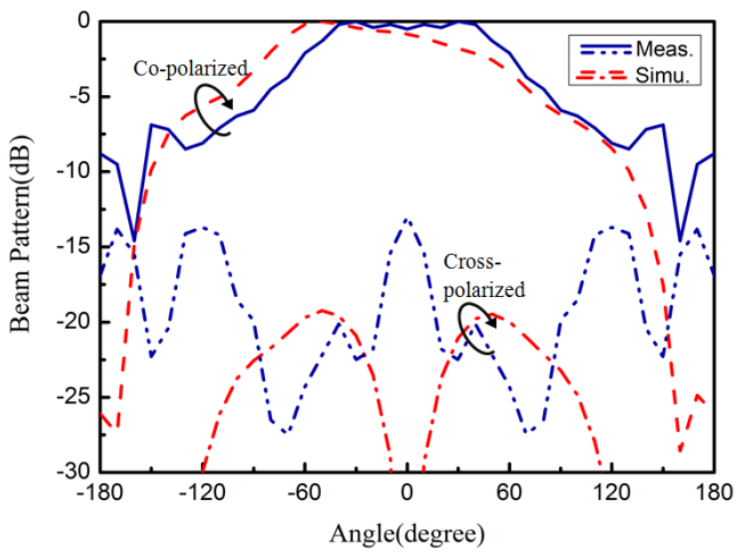

Fig. 7. Simulated and measured E-plane normalized radiation pattern of the single element antenna. 


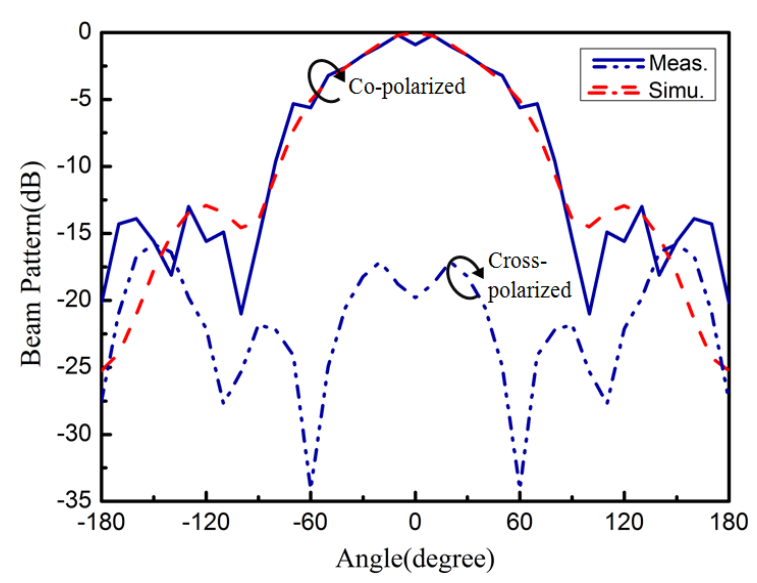

Fig. 8. Simulated and measured H-plane normalized radiation pattern of the single element antenna.

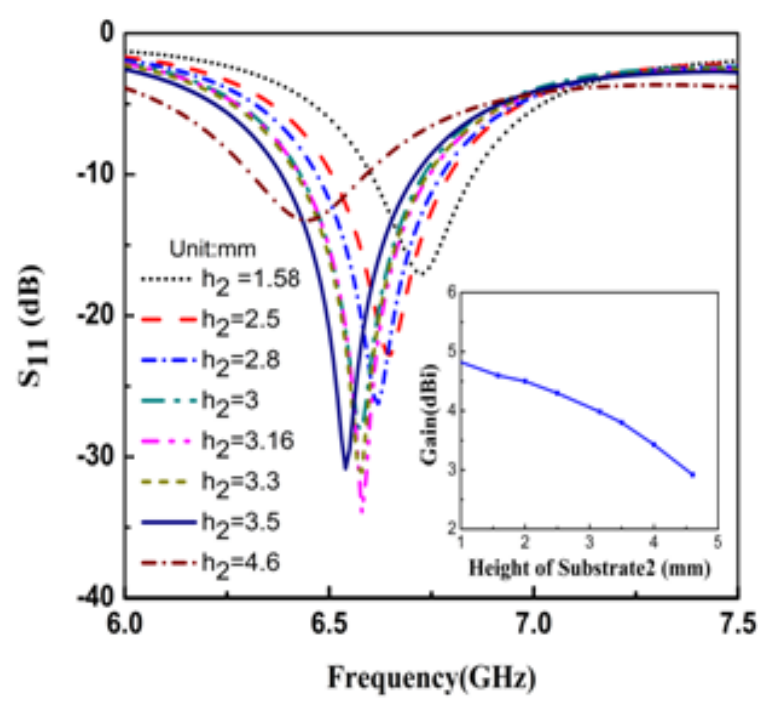

Fig. 9. Simulated $S_{11}$ of the DRA for different height of substrate2. Inset shows the corresponding gain plot.

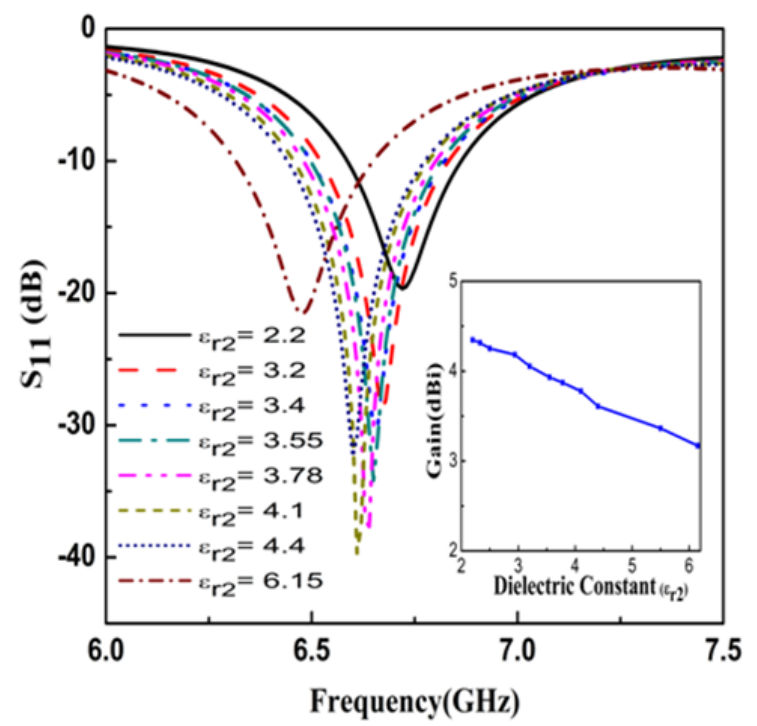

Fig. 10. Simulated $S_{11}$ of the DRA for different dielectric constant of substrate2. Inset shows the corresponding gain plot.

\section{Parameter Analysis}

\subsection{Effect of Height of Substrate2}

Figure 9 shows the variation of reflection coefficient with change in height of substrate $2 h_{2}$ and corresponding gain plot also depicted taking FR4 $\left(\varepsilon_{\mathrm{r} 2}=4.4\right)$ as substrate2. It can be seen that the height $h_{2}$ of substrate 2 has considerable effect on the reflection coefficient and gain of the antenna. If $h_{2}$ is become equal with the height of RDRA then the radiation of the antenna decreases significantly. The resonance frequency is shifted and matching performance also altered with the variation of $h_{2}$ due to the change in effective permittivity. After optimization we have consider FR4 of height $3.16 \mathrm{~mm}$ as substrate 2 since the best matching is achieved for $h_{2}=3.16 \mathrm{~mm}$.

\subsection{Effect of Dielectric Constant of Substrate 2}

Effect of dielectric constant of substrate 2 on operating frequency is also studied by keeping the height of substrate 2 fixed at $3.16 \mathrm{~mm}$. From the simulation results as shown in Fig. 10 it has been observed that operating frequency decrease with increase in $\varepsilon_{\mathrm{r} 2}$. The variation in the operating frequency mainly caused due to the change in effective permittivity surroundings of the RDRA. Radiation performance (gain) is also degraded with increase in $\varepsilon_{\mathrm{r} 2}$. Though the best matching performance is achieved for $\varepsilon_{\mathrm{r} 2}=4.1$ we have used FR4 of $\varepsilon_{\mathrm{r} 2}=4.4$ as substrate2. As FR4 of $\varepsilon_{\mathrm{r} 2}=4.4$ is easily available and it is more close to 4.1 so we have considered it as substrate 2 which gives good matching and desirable gain at the operating frequency.

\subsection{Study of Mutual Coupling}

The mutual coupling $\mathrm{S}_{21}$ between two elements is studied both for embedded and isolated DRA. Since we extent our work to design an array using the proposed method, the impact of substrate 2 on mutual coupling between two elements is also explored. The center-to-center

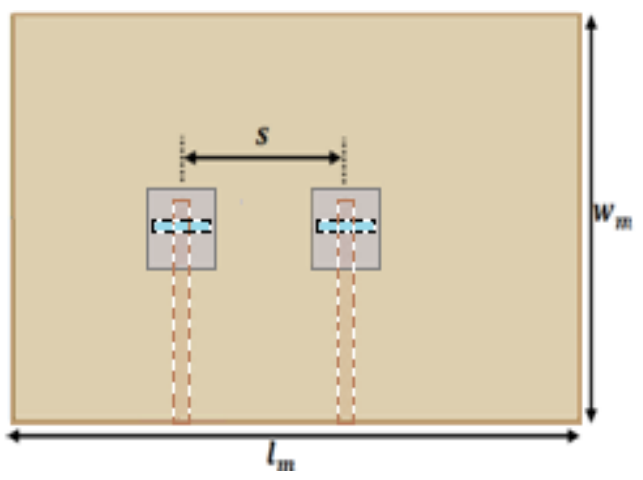

Fig. 11. Schematic diagram of two RDRA placed at distance $s$ on a substrate of length $l_{\mathrm{m}}=80 \mathrm{~mm}$ and width $w_{\mathrm{m}}=48 \mathrm{~mm}$. 


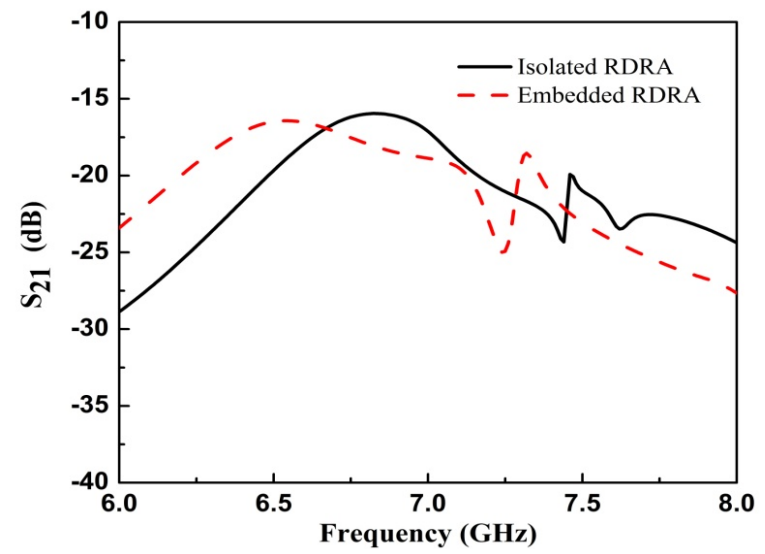

Fig. 12. Mutual coupling between two elements versus frequency with a center-to-center spacing of $0.5 \lambda_{\mathrm{o}}$ at $6.59 \mathrm{GHz}$ between the elements.

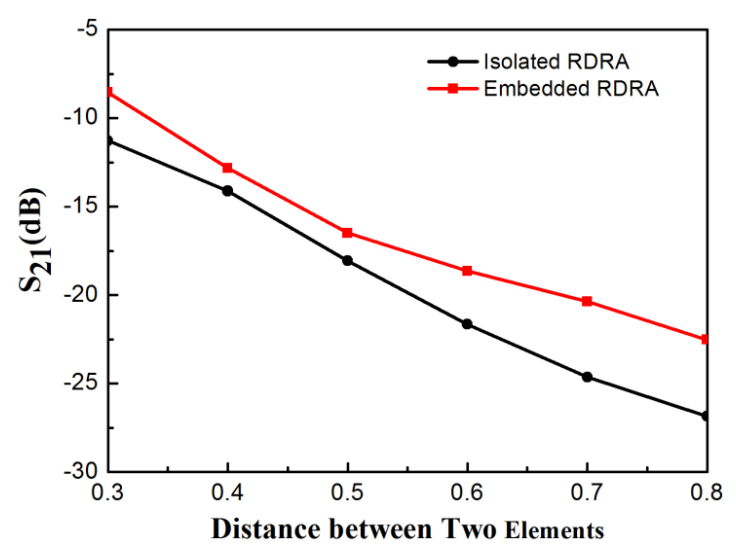

Fig. 13. Simulated mutual coupling versus center-to-center distance between two elements.

distance $s$ between two elements is varied from $0.3 \lambda_{\mathrm{o}}$ to $0.8 \lambda_{0}$. The variation of $\mathrm{S}_{21}$ with distance $s$ at resonance frequency for both isolated and embedded DRA is plotted in Fig. 13. Variation of $\mathrm{S}_{21}$ with frequency is shown in Fig. 12. From the simulation results, it is apparent that for embedded DRA $0.5 \lambda_{\mathrm{o}}$ separation between the two elements at $6.59 \mathrm{GHz}$, a $15-\mathrm{dB}$ coupling level is tolerable.

\section{Embedded RDRA Array}

\subsection{The Array Configuration}

In order to establish our concept of embedded DRA, we have designed a four-element array. The antenna configuration along with the feeding network is shown in Fig. 14. Response of the power divider is given in Fig. 15. Magnitude of $\mathrm{S}_{11}$ is well below $-10 \mathrm{~dB}$ within the frequency band of $5 \mathrm{GHz}$ to $8 \mathrm{GHz}$. Also we have found that all 4-ports are fed by equal amount of power within operating frequency band. The design parameters of the feeding network for array design are given in Tab. 1. Separation between the array elements is chosen as $c=d=23 \mathrm{~mm}$ to avoid mutual coupling effect by a reasonable amount (less than $-15 \mathrm{~dB})$.

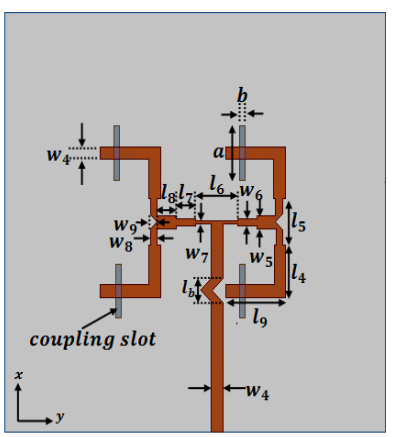

(a)

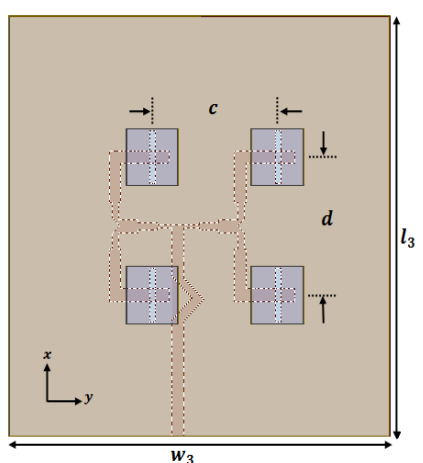

(b)
Fig. 14. Schematic diagram of $2 \times 2$ DRA array: (a) feed network below ground plane; (b) front view.

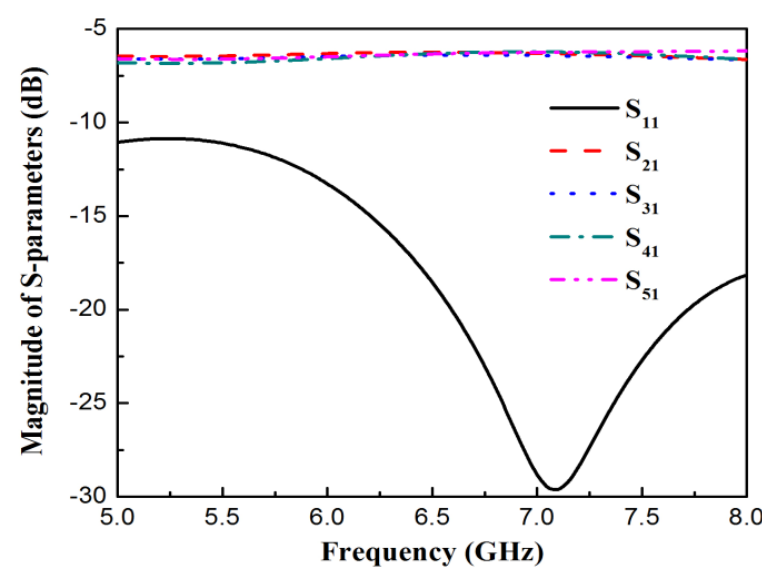

Fig. 15. Simulated S-parameters of the feeding network for the proposed $2 \times 2$ array.

\begin{tabular}{|c|c|c|c|}
\hline$w_{\mathbf{4}}=w_{\mathbf{5}}$ & $w_{\mathbf{6}}=\boldsymbol{w}_{\mathbf{8}}$ & $\boldsymbol{w}_{7}$ & $\boldsymbol{w}_{\mathbf{9}}$ \\
\hline 2.13 & 1.2 & 0.59 & 1.23 \\
\hline $\boldsymbol{l}_{\mathbf{4}}$ & $\boldsymbol{l}_{\mathbf{5}}=\boldsymbol{l}_{\mathbf{6}}$ & $\boldsymbol{l}_{\mathbf{7}}=\boldsymbol{l}_{\mathbf{8}}$ & $\boldsymbol{l}_{\mathbf{9}}$ \\
\hline 8.68 & 7.8 & 3.5 & 11 \\
\hline
\end{tabular}

Tab. 1. Dimension values of feeding network for array design (unit: $\mathrm{mm}$ ).

\subsection{Simulated and Measured Results}

The design parameters of the array are optimized by the full wave simulator HFSS. To validate the precision of the design, a prototype is fabricated and tested as illustrated in Fig. 16. The simulated and measured $S_{11}$ is depicted in Fig. 17. The measured resonant frequency of the proposed antenna array is $6.684 \mathrm{GHz}$ with $-10 \mathrm{~dB}$ impedance bandwidth of $4.94 \%$. A shift of $104 \mathrm{MHz}$ in frequency can be seen compared to the simulated one, which is presumably caused by the losses in feed network, change in the effective dielectric constant, the fabrication imperfection and assembling error. The far-field radiation patterns of the antenna array were measured in both planes with an Anritsu MG3692C signal generator as a source of continuous wave, standard gain horn antennas, and an Anritsu ML2437A power meter. The simulated and measured E- 
plane radiation pattern and $\mathrm{H}$-plane radiation pattern of the proposed embedded array are shown in Fig. 18 and Fig. 19, respectively.

A simulated gain of $11.1 \mathrm{dBi}$ is observed and measured gain of $10.4 \mathrm{dBi}$ is achieved for the E-plane radiation pattern of the array. For the H-plane radiation pattern, simulated and measured gains were $11.1 \mathrm{dBi}$ and $13.4 \mathrm{dBi}$, respectively. A good agreement between the simulated and measured radiation patterns for both E-plane and H-plane

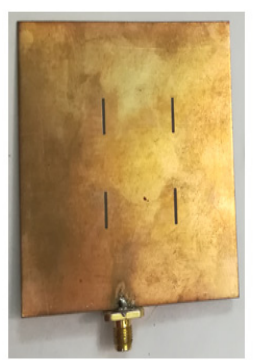

(a)

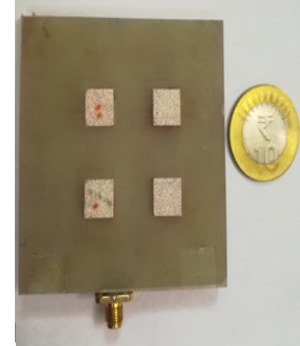

(b)

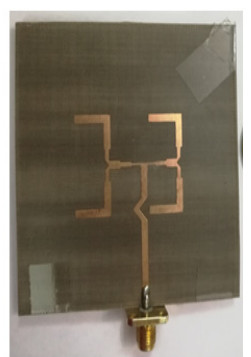

(c)
Fig. 16. Fabricated prototype of embedded RDRA array. (a) Perspective view of apertures on ground plane. (b) Perspective view of antenna array. (c) Perspective view of power divider network below the ground plane.

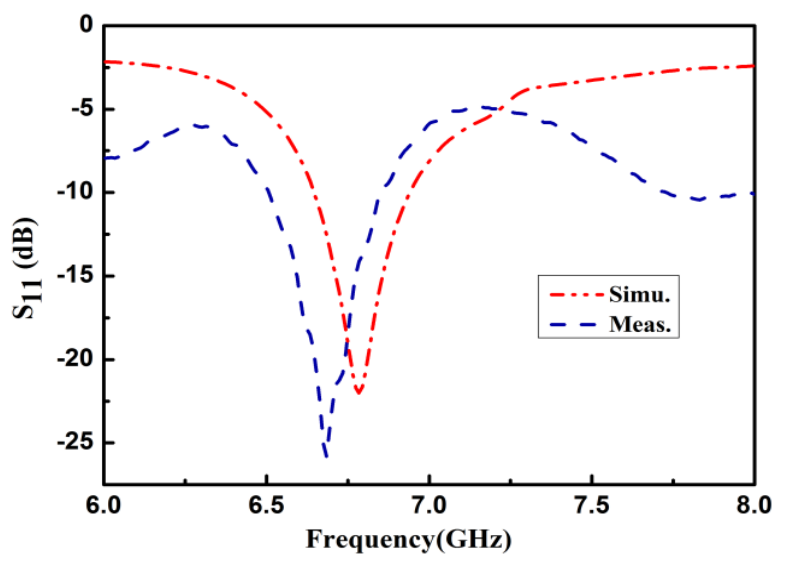

Fig. 17. Simulated and measured $S_{11}$ of the proposed antenna array. Parameters as in Tab. 1.

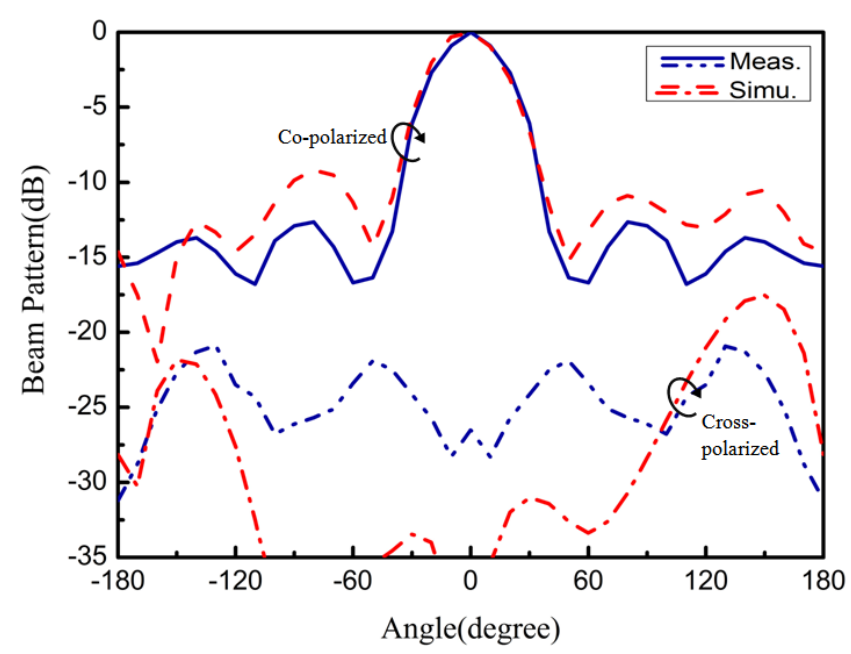

Fig. 18. Simulated and measured E-plane normalized radiation pattern of antenna array at $6.68 \mathrm{GHz}$.

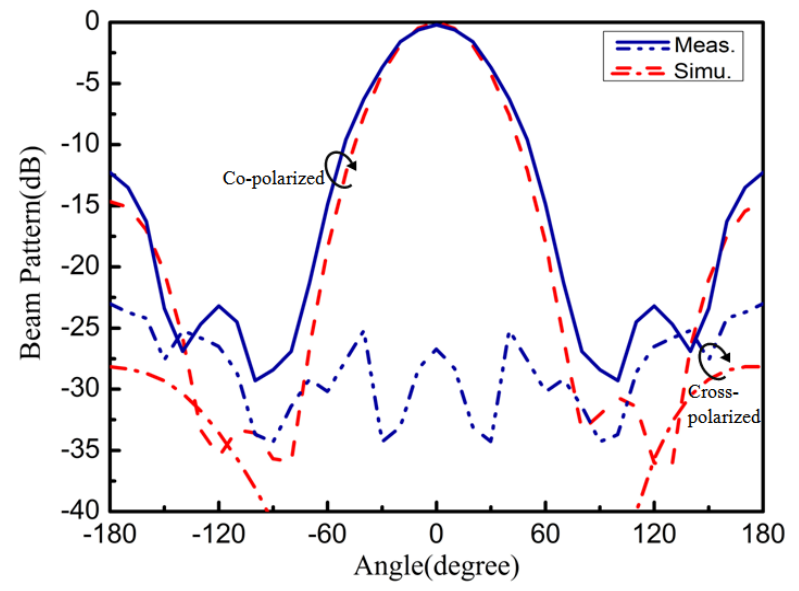

Fig. 19. Simulated and measured H-plane normalized radiation pattern of antenna array at $6.68 \mathrm{GHz}$.

is observed for the proposed antenna array. The $3-\mathrm{dB}$ beam-widths in the E- and H-planes are around $37^{\circ}$ and $62^{\circ}$. The co-cross polarized isolation levels are less than $-15 \mathrm{~dB}$ and the measured first sidelobe levels in E-plane are around $-15 \mathrm{~dB}$ whereas for $\mathrm{H}$-plane those are below $-17 \mathrm{~dB}$. Embedded slot feed array design helps to reduce cross-polarization level also significant reduction in sidelobe levels is observed.

\section{Conclusion}

With our proposed embedded design we can develop DRA blocks which become more rigid. For large array design using DRA, instead of mounting each individual element on the ground plane, from our work we can think of an inhomogeneous uneven substrate on which high permittivity regions are formed as per design requirement for efficient radiation. This makes whole arrangement become more stable and rigid in practice. A prototype of single element embedded RDRA has been fabricated and measured. The simulated results of reflection coefficients and radiation patterns have been found to be in reasonably good agreement with measured results. A prototype of $2 \times 2$ array is also fabricated and measured. The measured maximum gain of $13.4 \mathrm{dBi}$ has been found for the proposed DRA array. Embedded slot feed array design notably reduces cross-polarization level and also significant reduction in sidelobe levels is obtained. The proposed DRA array has very simple geometry and is inexpensive to fabricate. Due to non-availability of measurement facility we restrict our design within microwave frequency band. But the proposed DRA array is supposed to be a good approach in $\mathrm{mm}$ wave frequency band for $5 \mathrm{G}$ communication technology which is currently going through many challenges. The proposed antenna array has increased the reliability of use DRA for air/space born systems.

\section{Acknowledgments}

The authors thank senior colleagues for their valuable 
comments which have helped to improve the quality of paper.

\section{References}

[1] LUK, K. M., LEUNG, K. W. Dielectric Resonator Antennas. Baldock, Hertfordshire, England: Research Studies Press LTD, 2003. ISBN: $086380263 \mathrm{X}$

[2] PETOSA, A. Dielectric Resonator Antenna Handbook. Norwood, MA: Artech House, 2007. ISBN: 1596932066

[3] MONGIA, R. K., ITTIPIBOON, A. Theoretical and experimental investigations on rectangular dielectric resonator antennas. IEEE Transactions on Antennas and Propagation, 1997, vol. 45, no. 9, p. 1348-1356. DOI: 10.1109/8.623123

[4] KAJFEZ, D., GUILLON, P. Dielectric Resonators. Norwood, MA: Artech House, 1986. ISBN: 1-884932-05-3

[5] GLISSON, A. W., KAJFEZ, D., JAMES, J. Evaluation of modes in dielectric resonators using a surface a surface integral equation formulation. IEEE Transactions on Microwave Theory and Techniques, 1983, vol. 31, no. 12, p. 1023-1029. DOI: 10.1109/TMTT.1983.1131656

[6] KAJFEZ, D., GLISSON, A. W., JAMES, J. Computed modal field distributions for isolated dielectric resonators. IEEE Transactions on Microwave Theory and Techniques, 1984, vol. 32, no. 12, p. 1609-1616. DOI: 10.1109/TMTT.1984.1132900

[7] ANTAR, Y. M. M., FAN, Z. Theoretical investigation of aperture coupled rectangular dielectric resonator antenna. IEE ProceedingsMicrowave, Antennas and Propagation, 1996, vol. 143, no. 2, p. 113-118. DOI: 10.1049/ip-map:19960269

[8] PAN, Y. M., LEUNG, K. W., LUK, K. M. Design of the millimeter-wave rectangular dielectric resonator antenna using a higher-order mode. IEEE Transactions on Antennas and Propagation, 2011, vol. 59, no. 8, p. 2780-2788. DOI: 10.1109/TAP.2011.2158962

[9] NIKKHAH, M. R., KISHK, A. A., MOHASSEL, J. R. Wideband DRA array placed on array of slot windows. IEEE Transactions on Antennas and Propagation, 2015, vol. 63, no. 12, p. 5382-5390. DOI: 10.1109/TAP.2015.2490246

[10] LIN, J. H., SHEN, W., YANG, K. A low-sidelobe and wideband series-fed linear dielectric resonator antenna array. IEEE Antennas and Wireless Propagation Letters, 2017, vol. 16, p. 513-516. DOI: 10.1109/LAWP.2016.2586579

[11] RANA, B., PARUI, S. K. Nonresonant microstrip patch-fed dielectric resonator antenna array. IEEE Antennas and Wireless Propagation Letters, 2015, vol. 14, p. 747-750. DOI: 10.1109/LAWP.2014.2379624

[12] KISHK, A. A. Experimental study of broadband embedded dielectric resonator antennas excited by a narrow slot. IEEE Antennas and Wireless Propagation Letters, 2015, vol. 4, p. 79-81. DOI: 10.1109/LAWP.2005.844648
[13] SANGIOVANnI, A., DAUVIGNAC, J. Y., PICHOT, CH. Embedded dielectric resonator antenna for bandwidth enhancement. Electronics Letter, 1997, vol. 33, no. 25, p. 2090-2091. DOI: 10.1049/el:19971430

[14] PETOSA, A., THIRAKOUNE, S., ZULIANI, M., et al. Comparison between planar arrays of perforated DRAs and microstrip patches. In IEEE Antennas and Propagation Society International Symposium. Washington (USA), 2005, vol. 2. DOI: 10.1109/APS.2005.1551764

[15] PETOSA, A., THIRAKOUNE, S., ITTIPIBOON, A. Array of perforated dielectric resonator antennas. In IEEE Antennas and Propagation Society International Symposium. 2004, vol. 1 p. 1106-1109. DOI: 10.1109/APS.2004.1329868

[16] MOVAHEDINIA, R., CHAHARMIR, M. R., SEBAK, A. R., et al. Realization of large dielectric resonator antenna ESPAR. IEEE Transactions on Antennas and Propagation, 2017, vol. 65, no. 7, p. 3744-3749. DOI: 10.1109/TAP.2017.2705024

\section{About the Authors ...}

Suparna BALLAV received the B.Sc. (Physics Hons) degree in 2011 and the B.Tech. and M.Tech in Radio Physics and Electronics from the University of Calcutta, India in the year 2014 and 2016, respectively. She is pursuing Ph.D. degree in Microwave Engineering at the Dept. of Electronics and Tele-communication Engineering, Indian Institute of Engineering Science and Technology (IIEST), Shibpur, West Bengal, India since august 2016. Her research interests include dielectric resonators for microwave circuits and antenna application.

Susanta Kumar PARUI received the B.Sc. degree in Physics and B.Tech. degree in Radio Physics and Electronics from the University of Calcutta, India in the year 1987 and 1990, respectively and the Ph. D. degree in Microwave Engineering from the Bengal Engineering and Science University (presently known as the Indian Institute of Engineering Science and Technology), Shibpur, India. From 1993 to 2000, he was an Instrument Engineer in Process control Industries. Since 2000, he has been associated with the Dept. of Electronics and Tele-Communication Engineering, Indian Institute of Engineering Science \& Technology, Shibpur and presently holds the post of Associate Professor. He is the author of more than 60 papers in referred journals and conference proceedings. His research interests include planar circuits, antennas, SIW, DGS, EBG, FSS and metamaterials. Dr. Parui was awarded post doctoral fellowship from the Royal Academy of Engineering, U.K in the year 2009. 